\title{
Parity-time symmetry breaking in optically coupled semiconductor lasers
}

\author{
Joseph S. Suelzer ${ }^{\mathrm{a}, \mathrm{b}}$, Yogesh N. Joglekar ${ }^{\mathrm{b}}$, and Gautam Vemuri ${ }^{\mathrm{b}}$

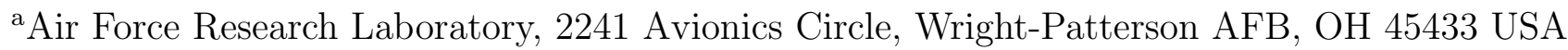 \\ ${ }^{b}$ Department of Physics, Indiana University Purdue University Indianapolis (IUPUI), $402 \mathrm{~N}$. \\ Blackford St., Indianapolis, USA
}

\begin{abstract}
We experimentally demonstrate the realization of a parity-time (PT) symmetry breaking in optically coupled semiconductor lasers (SCLs). The two SCLs are identical except for a detuning between their optical emission frequencies. This detuning is analogous to the gain-loss parameter found in optical PT systems. To model the coupled SCLs, we employ the standard rate equations describing the electric field and carrier inversion of each SCL, and show that, under certain conditions, the rate equations reduce to the canonical, two-site PTsymmetric model. This model captures the global behavior of the laser intensity as the system parameters are varied. Overall, we find that this bulk system (coupled SCLs) provides an excellent test-bed to probe the characteristics of PT-breaking transitions, including the effects of time delay.
\end{abstract}

Keywords: Non-Hermitian physics, coupled semiconductor lasers, nonlinear dynamics

\section{INTRODUCTION}

Carl Bender and Stefan Boettcher demonstrated that non-Hermitian Hamiltonians, invariant under the combined operations of parity $(\mathrm{P})$ and time-reversal $(\mathrm{T})$, may have a set of entirely real eigenvalues. ${ }^{1}$ In particular, the eigenvalues can undergo a transition from purely real to complex upon changing a parameter that controls the non-Hermiticity of the Hamiltonian. These surprising results sparked a theoretical interest in PT-symmetric systems. The implications of a non-Hermitian Hamiltonian on our understanding of quantum theory continue to be debated and studied in mathematical physics. ${ }^{2-6}$

Recently, the study of PT-symmetric Hamiltonians has evolved from a purely theoretical interest to an experimental realization, ${ }^{7-10}$ and is now showing promise in applied optics. ${ }^{11,12}$ This shift is due to two factors. The first is a well-known equivalence between the time-independent Schrodinger equation and the Helmholtz equation in optics, and second is the ability to implement complex, gain and loss potentials in the optical setting. ${ }^{13}$ Initially this equivalence was experimentally achieved by designing the optical system such the gain and loss of the material were balanced. ${ }^{7}$ By taking advantage of the ability to steer the system into a region of interest, i.e., a region where the symmetry is broken or unbroken, these investigations are paving the way toward applications in laser physics ${ }^{14-16}$ and synthetic photonic devices ${ }^{17}$ (see references therein).

A common challenge with these systems is the fabrication of devices with parameters required to ensure the PT-symmetry. We show that this challenge can be circumvented by using a bulk system, and demonstrate the ability to probe PT-symmetric features using two optically coupled, commercial semiconductor lasers (SCLs). The system we investigate has an intrinsic time-delay associated with the optically coupled fields, because of the finite distance $L$ between the two SCLs. In addition, we discuss the similarities and differences between a conventional PT-system (e.g. evanescently coupled waveguides) and optically coupled SCLs by comparing both the model and experimental observations.

To understand how these distinctions emerge, in Sec. 2 we introduce the full model comprising four coupled nonlinear rate equations that describe the electric field and carrier number inversion of each SCL. We show the remarkable feature that the rate equations reduce to a $2 \times 2 \mathrm{PT}$-model. The experimental procedure is outlined

Further author information: (Send correspondence to JSS)

JSS: E-mail: joseph.suelzer.ctr@us.af.mil, Telephone: 19377138712

Active Photonic Materials VIII, edited by Ganapathi S. Subramania,

Stavroula Foteinopoulou, Proc. of SPIE Vol. 9920, 99201M - (c) 2016

SPIE · CCC code: $0277-786$ X/16/\$18 $\cdot$ doi: $10.1117 / 12.2239487$

Proc. of SPIE Vol. $992099201 \mathrm{M}-1$ 
in Sec. 3. In Sec. 4, we show the excellent agreement with the simulations produced by the full rate equations. and we analyze the experimental observations by comparing them with the $2 \times 2$ PT-model and the simulations. The conclusions are outlined in Sec. 5. We highlight that the PT-symmetry breaking features are captured using bulk components, i.e., off-the-shelf SCLs.

\section{RATE EQUATIONS AND THE $2 \times 2$ PT-MODEL}

We model the system of two optically coupled lasers using an extension of the well known Lang-Koboyashi rate equations which describe a single semiconductor laser subject to optical feedback. ${ }^{18}$ The coupled SCL model we employ assumes that the SCLs are identical, except for their free-running emission frequencies (at threshold), and operate at nearly identical optical frequencies $\left(\omega_{1} \approx \omega_{2}\right) \cdot{ }^{19}$ The slowly varying electric fields are defined in a symmetric reference frame, characterized by $\theta=\left(\omega_{1}+\omega_{2}\right) / 2$. The rate equations describing the normalized complex electric fields $E_{1,2}$ and the normalized excess carrier-densities $N_{1,2}$ are as follows:

$$
\begin{aligned}
\frac{d E_{1}}{d t} & =(1+i \alpha) N_{1}(t) E_{1}(t)+i \Delta \omega E_{1}(t)+\kappa e^{-i \theta \tau} E_{2}(t-\tau), \\
\frac{d E_{2}}{d t} & =(1+i \alpha) N_{2}(t) E_{2}(t)-i \Delta \omega E_{2}(t)+\kappa e^{-i \theta \tau} E_{1}(t-\tau), \\
T \frac{d N_{1}}{d t} & =J_{1}-N_{1}(t)-\left(1+2 N_{1}(t)\right)\left|E_{1}(t)\right|^{2}, \\
T \frac{d N_{2}}{d t} & =J_{2}-N_{2}(t)-\left(1+2 N_{2}(t)\right)\left|E_{2}(t)\right|^{2},
\end{aligned}
$$

where $\alpha \sim 2-10$ is the semiconductor-dependent linewidth enhancement factor, $\tau$ is the time-delay in the coupling, $J_{1,2} \propto I_{1,2} / I_{\text {thr }}$ is the pumping current above threshold, and $T=\tau_{s} / \tau_{p}$ is the ratio of the carrier lifetime $\tau_{s}$ to the photon lifetime $\tau_{p}$. The two system parameters that permit exploration of the PT-breaking transition, $\kappa$ and $\Delta \omega=\left(\omega_{1}-\omega_{2}\right) / 2$, are the coupling strength and frequency detuning, respectively. These are examined in more detail when we outline the experimental procedure in Sec. 3. In this way, it will become clear how we experimentally determine their numerical values for the PT-model and simulations.

The parameter values used in our simulations are defined in Table 1. The stated values correspond to those typical of the SCL used in our experiment. ${ }^{20}$ We refer to Eqns. (1) as the full-model in order to distinguish them from the simplified PT-model. Given the high degree of complexity and nonlinearity of this system it is often necessary to integrate the rate equations of the full-model (Eqns, (1)) in order to properly capture the dynamics. To that end, we use a modified fourth order Runge-Kutta method with a time-step size that varies from $0.1 \mathrm{ps}$ to $10 \mathrm{ps}$.

Table 1. The parameter values for a typical SCL that are used in the simulations.

\begin{tabular}{lll}
\hline Quantity & Symbol & Value \\
\hline Linewidth enhancement factor & $\alpha$ & 5 \\
Coupling strength & $\kappa$ & $0-0.2$ \\
Delay-time & $\tau$ & $0-500$ \\
Ratio of carrier to photon lifetime & $T$ & 100 \\
Pump rate & $J$ & $0.01-0.10$ \\
\hline
\end{tabular}

Although the full model of rate equations is complicated, for judicious choice of parameters, it is simplified substantially. When the excess carrier-density is small, i.e., $N_{1,2}(t) \rightarrow 0$, and the time-delay is negligible, the equations for the time evolution of the electric fields become

$$
\frac{d}{d t}\left[\begin{array}{l}
E_{1}(t) \\
E_{2}(t)
\end{array}\right]=\left(\begin{array}{cc}
i \Delta \omega & \kappa \\
\kappa & -i \Delta \omega
\end{array}\right)\left[\begin{array}{l}
E_{1}(t) \\
E_{2}(t)
\end{array}\right]=M\left[\begin{array}{l}
E_{1}(t) \\
E_{2}(t)
\end{array}\right] .
$$

Equation (2) is reminiscent of the Schrodinger equation in quantum theory, but with two important differences. The first is the absence of an $i$ on the left-hand side of the equation. The second is that in contrast to the conventional quantum theory, the matrix $M$ is not Hermitian (or anti-Hermitian), i.e., $M \neq M^{\dagger}$ and $(i M) \neq$ 
$(i M)^{\dagger}$. One can show that the matrix $i M$ is invariant under the combined operations of parity $\mathrm{P}=\sigma_{z}$ and time reversal $\mathrm{T}=*$, where $\sigma_{z}$ is the z-Pauli matrix and $*$ denotes complex conjugation. We point out that the Hermitian coupling term in conventional PT systems is now replaced by $\pm i \Delta \omega$, the anti-Hermitian detuning term which accounts for frequency pulling that is typical of coupled laser oscillators operating at different frequencies. ${ }^{21}$

It is straightforward to show that the eigenvalues of Eqn. (2) are $\lambda_{1,2}= \pm \sqrt{\kappa^{2}-\Delta \omega^{2}}$. Thus they are real when $k \geq \Delta \omega$ and become purely imaginary when $k<\Delta \omega$. By examining the eigenvalues along with the form of the solution $\left(e^{-\lambda t}\right)$, one can anticipate the behavior of the system across a PT-transition that occurs at $\kappa=\Delta \omega$. Below the threshold $(\kappa>\Delta \omega)$, the amplitudes exponentially grow or decay and above threshold $(\kappa<\Delta \omega)$ the amplitudes undergo oscillations. We remark, however, that exponential growth of the field cannot occur indefinitely since the inversion of the laser will eventually be depleted as photons are added to the cavity. Therefore, we expect the PT-model to accurately describe the lasers behavior near or above the PT-transition. To see how the PT parameters are controlled, it is important to examine the experimental design in more detail.

\section{EXPERIMENTAL DESIGN}

Figure 1 is a schematic of the experimental configuration consisting of two single mode (HL7851G) semiconductor lasers (SCL1 and SCL2), an external cavity consisting of two beam-splitting mirrors (BS1 and BS2) which optically couples the two SCLs, and an external control of the coupling strength $\kappa$ via the variable neutral density filter (VND). The SCLs are identical, except for their free-running optical frequencies and threshold pump currents. The transmission through the VND is determined by an independent laser (L3) and photodiode

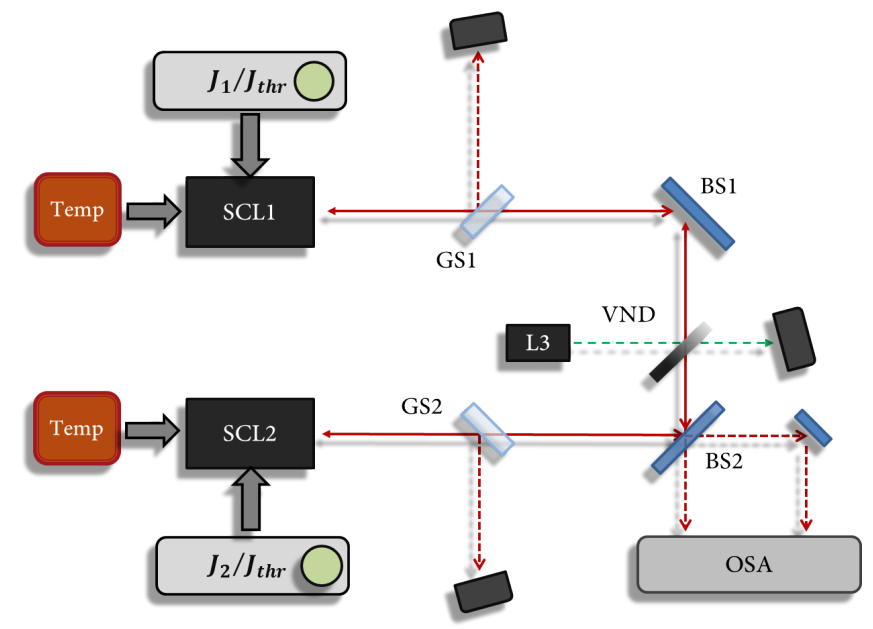

Figure 1. Schmatic of the experimental set-up. SCL: semiconductor laser, GS: glass slide, M: mirror, VND: variable neutral density filter (mounted on a translation stage), L3: laser used to quantify experimental coupling strength, PD: photodiodes, $P_{1,2}$ : pump-current controller.

(PD3) which allows us to calibrate the experimental and theoretical coupling strength, $\kappa \equiv\left(1-r^{2}\right) /\left(r \tau_{\text {in }}\right) \xi \tau_{p}$, where $r$ is the reflectivity of the external laser facet, $\xi^{2}$ is the fraction of optical power transmitted by all the optical elements, $\tau_{\text {in }}$ is the internal laser round-trip time, and $\tau_{p}$ is the photon lifetime. Once the transmission through the VND is recorded, $\xi^{2}$ can be determined since all the other optical elements are fixed.

This model assumes that the fractional power is entirely coupled into the active region of the SCL. However, Cheng et al. showed that the measured fractional power $(\kappa)$ needed to be reduced by a factor of 10 in order to accurately describe the dynamics ${ }^{20}$ from this model. Hence, the coupling strength $(\kappa)$ used in the simulations is a free parameter (within the bounds mentioned by $\mathrm{Cheng}^{20}$ ). The experiment is designed such that the coupling strengths to the two lasers are equal, and a Faraday-rotator (not shown) is placed in the coupling beam path to ensure that self-coupling is eliminated. The glass slides (GS1 and GS2) independently reflect a portion (8\%) of the intensity from SCL1 and SCL2, and the measurement of the laser intensities is performed using two $1 \mathrm{GHz}$ 
photo-diodes (PD1 and PD2) in conjunction with a $1 \mathrm{GHz}$ oscilloscope. The current and temperature of the lasers are stabilized to an accuracy of $0.01 \mathrm{~mA}$ and $0.01 \mathrm{C}$, respectively.

\section{RESULTS}

The procedure we employ allows us to determine the parameter space wherein the PT-model can accurately describes the experimental observations. This is achieved by scanning the pump current which induces a change in the emitted laser intensity and optical frequency. The dependence of the intensity and frequency on the adjusted pump current $(\Delta P)$, is given by Eqns. (3),

$$
\begin{aligned}
\omega(\Delta P) & =\omega_{0}-k \Delta P, \\
I(\Delta P) & =I_{\mathrm{thr}}+\eta_{s} \Delta P,
\end{aligned}
$$

where $\omega_{0}$ is the laser frequency at the threshold, and $\Delta P$ is the pump-current with the threshold pump current subtracted. The slopes $k$ and $e t a_{s}$ are intrinsic characteristics of the SCL, and were determined to be $k=1.84$ $\mathrm{GHz} / \mathrm{mA}$ and $\eta_{s}=0.55 \mathrm{~mW} / \mathrm{mA}$. The variations in the optical frequency were a desired effect since, ultimately, we wanted to control the frequency difference $\Delta \omega$ between the two lasers.

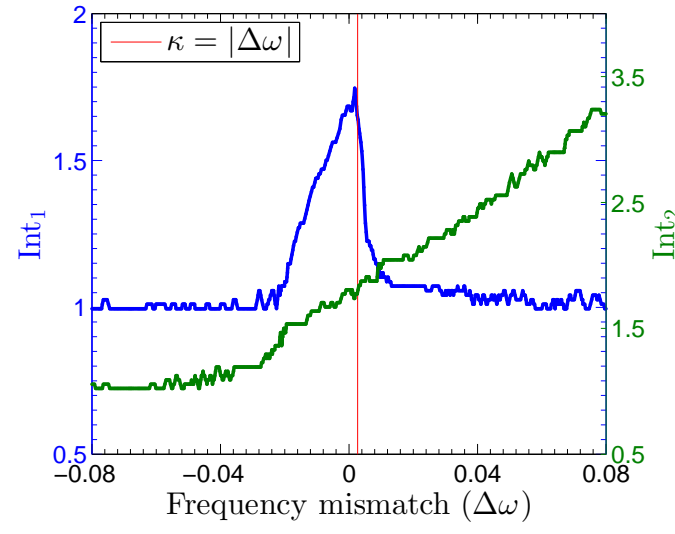

(a)

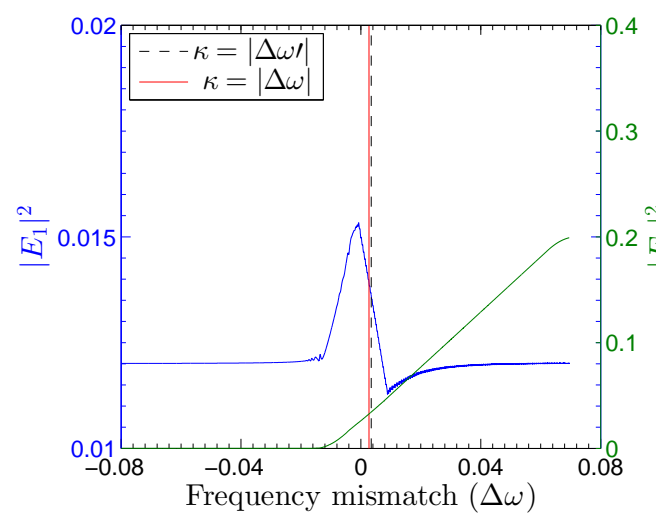

(c)

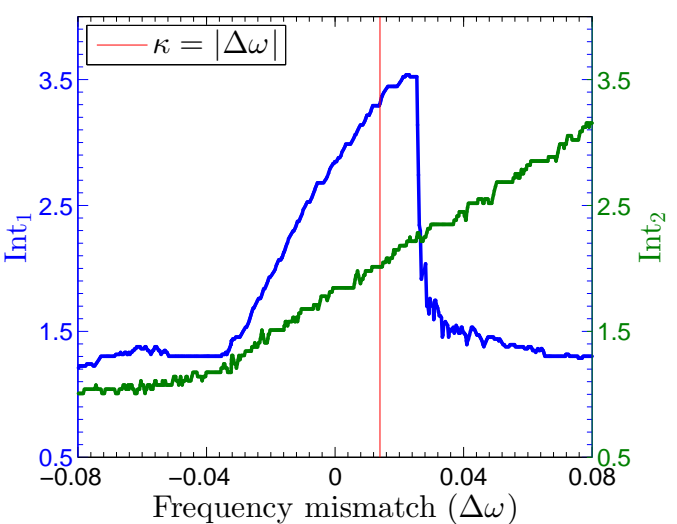

(b)

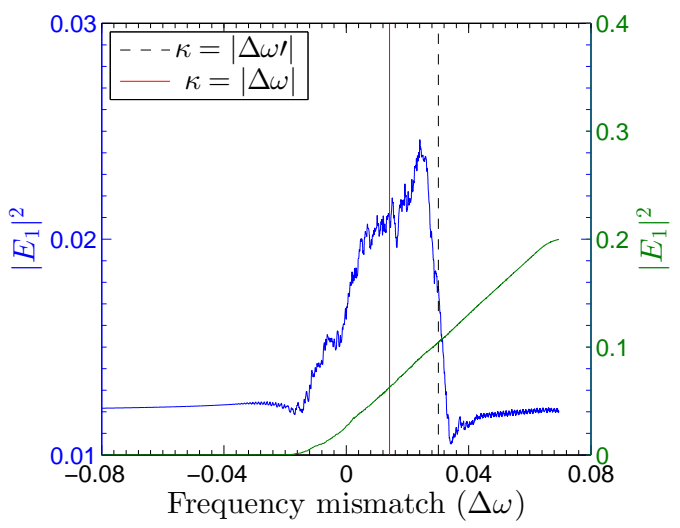

(d)

Figure 2. Experimental (a,b) and numerical (c,d) intensities of SCL1 (blue) and SCL2 (green) when the coupling strength is $\kappa=0.0027(\mathrm{a}, \mathrm{c})$ and $\kappa=0.014(\mathrm{~b}, \mathrm{~d})$. The pump current $P_{2}$ is decreased from $P_{2}=1.3$ to $P_{2}=0.8$. The vertical lines indicate the locations when $\Delta \omega=\kappa$ (black dashed) and when the phase breaking occurs $\dot{\phi}(t)=\Delta \omega^{\prime} \neq 0$ (red solid).

Figure 2 shows a set of typical results - experimental and simulations - for two different coupling strengths of (a) $\kappa=0.0027$ and (b) $\kappa=0.014$. In Fig. 2, SCL1 is operated at $2 \%$ above threshold and SCL2 is initially at 


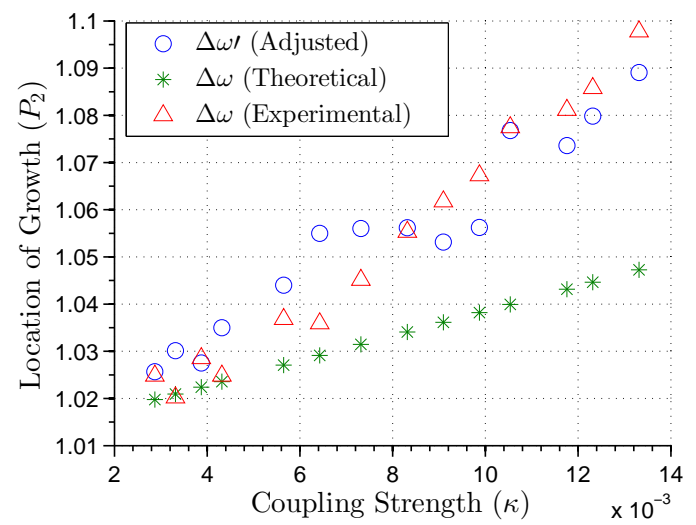

Figure 3. Experimental plot of the pump current value where the onset of growth begin for increasing coupling strength $\kappa$ values, which are marked with red diamonds. The blue circles correspond to the simulated location where phase locking is broken $\left(d \phi_{1} / d t \neq d \phi_{2} / d t\right)$. The green stars represent the PT-transition boundary predicted by the simple $2 \times 2$ model $(\kappa=|\Delta \omega|)$.

$30 \%$ above threshold. The frequency detuning is large compared to the coupling strength $(|\Delta \omega|>\kappa)$. The pump current to SCL2 is then decreased slowly (at $<10 \mathrm{~Hz}$ ) from $P_{2}=1.3$ to $P_{2}=0.8$, consequently decreasing the frequency mismatch $\Delta \omega$. Examining Fig. 2 , it is clear that as $P_{2}$ is decreased, the intensity of SCL2, Int 2 (green) decreases and the intensity of SCL1, Int 1 (blue) remains constant until a critical pump current is reached when a sudden growth of intensity occurs. This abrupt increase in intensity marks the PT transition threshold. We compare this threshold with the phase-breaking threshold, shown in Fig. 2(c) and 2(d) with the black dashed line. To obtain the latter, we calculated the phases $\phi_{1,2}(t)$ which are extracted from the complex electric fields $E_{1,2}(t)$, evaluated their time derivatives, and obtained the frequency mismatch at which their difference is no longer zero, i.e., $d \phi_{1} / d t \neq d \phi_{2} / d t$. The simulations agreed well with the experiments shown in Fig. 2(a).

Figure 3 shows the results from the full model, predictions of the simplified $2 \times 2$ PT-model, and experimental data. It is clear that the agreement is good at low coupling strengths, but at higher coupling strengths, the simplified model departs significantly from the experiments and the predictions of the full model. The dependence of the laser intensity on the pump current subsequently determines the parameter window where the simplified PT-model breaks down. A cause of the breakdown is the requirement that the intensities of the two SCLs need to be approximately equal $\left(I_{1} \approx I_{2}\right)$ near the PT-threshold $(\kappa=|\Delta \omega|)$. Because of the coupled nature between the number of photons (intensity) inside the laser cavity and the inversion, an inequality between the intensities, $I_{1}$ and $I_{2}$, leads to an inequality in the inversions of each laser (i.e. $N_{1} \neq N_{2}$ ). If the difference between the inversions is too large then the full-model can longer reduce to the simplified PT-model because the diagonal terms in the matrix representation are no longer equal. It is, however, remarkable, that the full model contains within it the PT-symmetric behavior information despite the full model not being the typical PT-symmetric model.

Another method we use to examine the PT transition is to follow the evolution of the eigenvalues of the rate equation. In the presence of non-zero inversion, i.e., $N_{1,2}=(N \pm \Delta N) / 2 \neq 0$, diagonal elements of the matrix $M$ in Eq.(2) are modified. To obtain the eigenvalues $\lambda$ defined by Eqn.(4),

$$
\lambda^{2}+\Delta \omega^{2}-\kappa^{2}-\lambda N(1+i \alpha)+\frac{1}{2}\left(N^{2}-\Delta N^{2}\right)(1+i \alpha)^{2}-i \Delta \omega \Delta N(1+i \alpha)=0,
$$

the full set of rate equations was solved as $J_{2}$ was scanned from $1.3 J_{\text {thr }}$ to $0.8 J_{\text {thr }}$ and the array of $N_{1,2}$ values were stored. These values of $N_{1,2}$ were then inserted into Eqn. (4). At each step of $P_{2}\left(N_{1,2}\right)$, the complex eigenvalues are determined and stored. After scanning the pump current $P_{2}$, a complete map of the eigenvalues is produced, shown in Fig. 4, which displays both eigenvalues $\lambda_{1,2}$ in the complex plane. Figure 4(a) shows the eigenvalues when the feedback strength is $\kappa=0.0027$, and the color spectrum along each step corresponds to the pump current $P_{2}\left(N_{1,2}\right)$ at that location. We start by examining the evolution of each eigenvalue $\lambda_{1,2}$ individually 
shown in Fig. 4. We remind the reader that this figure represents the eigenvalues-movement for simulated profiles shown in Fig. 2(c) and Fig. 2(d).

Initially $\lambda_{1}$ (Fig. 4(a)) is purely imaginary ( $\Im \lambda_{1} \approx 0.01$ and $\Re \lambda_{1} \approx 0$ ), which corresponds to the oscillatory behavior of the intensity. Since we are taking a time average, these oscillations are smoothed out resulting in the steady-state behavior shown in Fig. 2 . As the pump current decreases, $\Im \lambda_{1}$ decreases while the real part remains zero $\left(\Re \lambda_{1} \approx 0\right)$. At the critical value when $\kappa=|\Delta \omega|, \lambda_{1}$ becomes real and $\Re \lambda_{1}$ suddenly increases while the imaginary component is negligible $\left(\Im \lambda_{1} \approx 0\right)$, which corresponds to the abrupt growth in the intensity. At this point, the PT-symmetry is broken because the carrier densities become non-negligible. One sees this by continuing to trace this evolution, where $\Re \lambda_{1}$ becomes negative and eventually returns to zero $\left(\Re \lambda_{1}=0\right)$.

Turning our attention to Fig. 4(b), which is produced when the coupling strength is $\kappa=0.014$, it is clear that the avoided crossing is still present, however its location is slightly shifted from the PT-breaking transition $\left(\lambda_{1,2}=0\right)$. The abrupt change in $\lambda_{2}$, shown in Fig. 4(b), occurs at a complex value, i.e., $\lambda_{2}$ has a non-zero real component, rather than a purely imaginary value. This highlights our observation that the PT-symmetric model (Eq. (2)) is valid for small feedback strengths. The shift in the avoided crossing location from the PT-symmetric case is a result of the non-negligible carrier densities, $N_{1,2}>0$. Since the coupling strength is large, the expected intensity growth should occur at a larger pump current $P_{2}$. The larger values of $P_{2}$ and $\kappa$ creates an asymmetry between the carrier densities $\left(N_{1} \neq N_{2}\right)$ and $N_{1}>0$. Therefore, the approximations used to obtain the simplified model are no longer valid and Eq. (1) no longer reduces to the PT model. However, it is interesting to note that even at larger coupling strengths $(\kappa>0.004)$, the avoided crossing continues to be present.

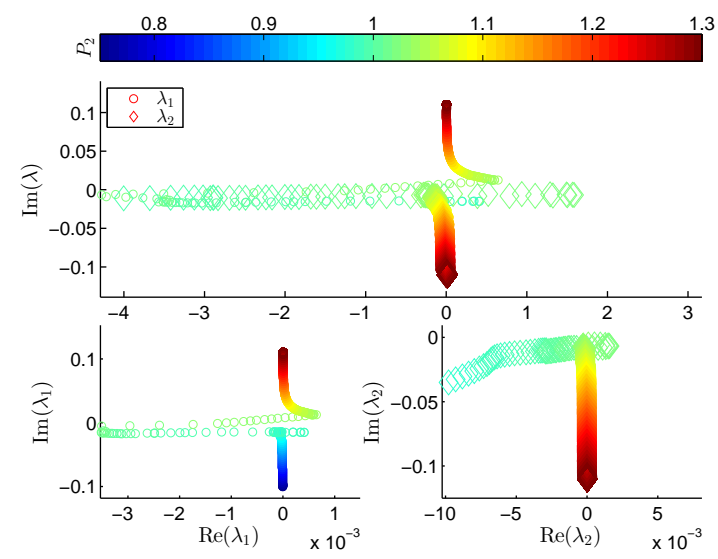

(a)

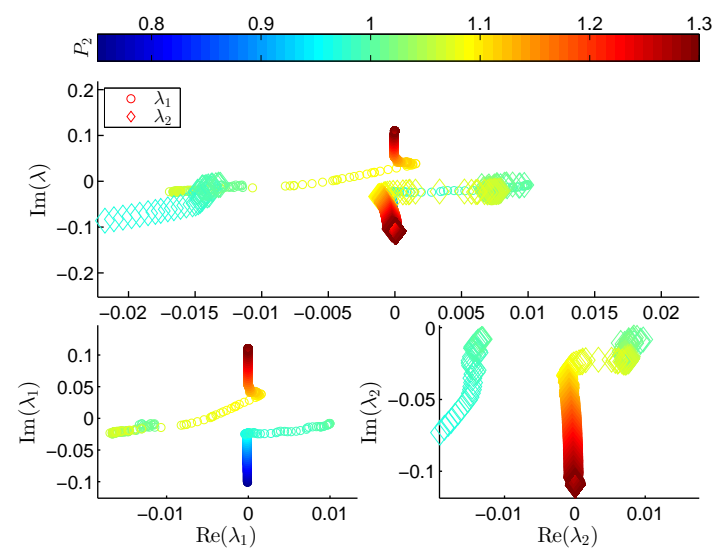

(b)

Figure 4. Evolution of eigenvalues for the two coupling strength $\kappa=0.003$ (a) and $\kappa=0.017$ (b) with same parameters as Fig. 2. The color profile corresponds to the pump current $P_{2}$ which is directly proportional to the frequency mismatch $\Delta \omega$. Each eigenvalue, $\lambda_{1}$ and $\lambda_{2}$, is shown separately in $(\mathrm{a} 2, \mathrm{~b} 2)$ and $(\mathrm{a} 3, \mathrm{~b} 3)$, respectively, and their superposition is shown in (a1,b1). Note that the avoided crossing in (a), which occurs for a pump current of $P_{1} \approx 1.05$, occurs at $\lambda \approx 0$ and indicates PT-transition.

\section{CONCLUSIONS}

In this paper, we demonstrated the realization of PT-symmetry breaking transition in optically coupled SCLs. In addition to this system comprising commercially available components, an advantage of coupled SCLs is the ability to easily tune the PT-transition parameters, which are the coupling strength $\kappa$ between the SCLs and the frequency detuning $\Delta \omega$. One can control these parameters by externally attenuating the coupled optical power (via a neutral density filter) and modifying the pump current (or temperature), respectively. We showed the remarkable feature that the standard rate equations describing coupled SCLs can reduce to a simplified $2 \times 2$ PT model. To capture the PT-transition, we compared the movement of the eigenvalues of the modified $2 \times 2$ model with the experimental and simulated intensities of the SCLs. This method allowed us to determine the 
parameter window wherein the PT-model accurately captures the global behavior. For small coupling strengths, the onset of intensity growth corresponded to the theoretical PT-transition. However, the deviation from the predicted value grew as the coupling strength became larger. This is understood by noting the increase pump current necessary for a PT-transition causes a large inequality between the carriers of the two SCLs. This inequality invalidates the assumptions made to arrive at the simplified PT-model. A method that circumvents this inequality is to vary the frequency detuning via the temperature, which we are currently exploring.

This system offers many avenues for future investigations. In particular, we plan to continue experimental investigations of the PT-transition by varying the temperature to one or both SCLs and by introducing substantial time-delay in the feedback. In this way, the effects of the time-delay on the PT-transition are manifested. This requires a modification of the PT model. Because of this modification, a question emerges whether the system still retains PT signatures in the presence of a time-delay. Another avenue is the ability to control the phase $\theta \tau$ of coupling term $\kappa$ which is present in the full-model. If the phase is incorporated, then this further modifies the PT model and allows tunability over the Hermicity of the PT matrix via the off-diagonal term. Overall, we are convinced that this system offers a unique test-bed to explore PT-symmetric systems.

\section{ACKNOWLEDGMENTS}

Part of this research was performed while JSS held an NRC Research Associateship award at the Air Force Research Laboratory, and JSS acknowledges AFOSR 16RYCOR332. This work was supported by NSF Grant no. DMR-1054020 (YJ). The views and opinions expressed in this paper (88ABW-2016-3789) are those of the authors and do not reflect the official policy or position of the United States Air Force, Department of Defense or the U.S. Government.

\section{REFERENCES}

[1] Bender, C. M. and Boettcher, S., "Real spectra in non-hermitian hamiltonians having pt symmetry," Phys. Rev. Lett. 80, 5243-5246 (Jun 1998).

[2] Bender, C. M., Brody, D. C., and Jones, H. F., "Complex extension of quantum mechanics," Phys. Rev. Lett. 89, 270401 (Dec 2002).

[3] Bender, C. M., Hook, D. W., Meisinger, P. N., and Wang, Q.-h., "Complex correspondence principle," Phys. Rev. Lett. 104, 061601 (Feb 2010).

[4] Scolarici, G. and Solombrino, L., "On the pseudo-hermitian nondiagonalizable hamiltonians," Journal of Mathematical Physics 44(10) (2003).

[5] Brody, D. C., "Consistency of pt-symmetric quantum mechanics," Journal of Physics A: Mathematical and Theoretical 49(10), 10LT03 (2016).

[6] Mostafazadeh, A., "Pseudo-hermiticity versus pt symmetry: The necessary condition for the reality of the spectrum of a non-hermitian hamiltonian," Journal of Mathematical Physics 43(1) (2002).

[7] Rüter, C. E., Makris, K. G., El-Ganainy, R., Christodoulides, D. N., Segev, M., and Kip, D., "Observation of parity-time symmetry in optics," Nature Physics 6(3), 192-195 (2010).

[8] Kottos, T., "Optical physics: Broken symmetry makes light work," Nature Physics 6(3), 166-167 (2010).

[9] Regensburger, A., Bersch, C., Miri, M.-A., Onishchukov, G., Christodoulides, D. N., and Peschel, U., "Parity-time synthetic photonic lattices," Nature 488(7410), 167-171 (2012).

[10] Cole, J. T., Makris, K. G., Musslimani, Z. H., Christodoulides, D. N., and Rotter, S., "Twofold $\mathcal{P} \mathcal{T}$ symmetry in doubly exponential optical lattices," Phys. Rev. A 93, 013803 (Jan 2016).

[11] Wimmer, M., Regensburger, A., Miri, M.-A., Bersch, C., Christodoulides, D. N., and Peschel, U., "Observation of optical solitons in pt-symmetric lattices," Nature communications 6 (2015).

[12] Rivolta, N. X. A. and Maes, B., "Symmetry recovery for coupled photonic modes with transversal pt symmetry," Opt. Lett. 40, 3922-3925 (Aug 2015).

[13] Zyablovsky, A. A., Vinogradov, A. P., Pukhov, A. A., Dorofeenko, A. V., and Lisyansky, A. A., "Ptsymmetry in optics," Physics-Uspekhi 57(11), 1063 (2014).

[14] Feng, L., Wong, Z. J., Ma, R.-M., Wang, Y., and Zhang, X., "Single-mode laser by parity-time symmetry breaking," Science 346(6212), 972-975 (2014). 
[15] Jing, H., Özdemir, S. K., Lü, X.-Y., Zhang, J., Yang, L., and Nori, F., "P T-symmetric phonon laser," Phys. Rev. Lett. 113, 053604 (Jul 2014).

[16] Hodaei, H., Miri, M.-A., Heinrich, M., Christodoulides, D. N., and Khajavikhan, M., "Paritytime-symmetric microring lasers," Science 346(6212), 975-978 (2014).

[17] Yidong, C., "Nonlinear optics: asymmetry from symmetry," Nature Physics 10(5), 336-337 (2014).

[18] Lang, R. and Kobayashi, K., "External optical feedback effects on semiconductor injection laser properties," IEEE Journal of Quantum Electronics 16, 347-355 (Mar 1980).

[19] Mulet, J., Masoller, C., and Mirasso, C. R., "Modeling bidirectionally coupled single-mode semiconductor lasers," Physical Review A 65(6), 063815 (2002).

[20] Takeuchi, Y., Shogenji, R., and Ohtsubo, J., "Chaotic dynamics in semiconductor lasers subjected to polarization-rotated optical feedback," Applied Physics Letters 93(18) (2008).

[21] Fader, W., "Theory of two coupled lasers," IEEE Journal of Quantum Electronics 21, 1838-1844 (Nov 1985). 